\title{
High frequency electron spin resonance in $\mathrm{Er}_{m} @ \mathrm{C}_{2 n}$
}

\author{
M.E.J. Boonman ${ }^{\mathrm{a}, *}$, P.H.M. van Loosdrecht ${ }^{\mathrm{b}}$, D.S. Bethune ${ }^{\mathrm{b}}$, I. Holleman ${ }^{\mathrm{a}}$, \\ G.J.M. Meijer ${ }^{\mathrm{a}}$, P.J.M. van Bentum ${ }^{\mathrm{a}}$ \\ ${ }^{a}$ High Field Magnet Laboratory and Research Institute for Materials, University of Nijmegen, Toernooiveld l, \\ NL-6525 ED Nijmegen. The Netherlands \\ ${ }^{\mathrm{b}}$ IBM Research Division, Almaden Research Center, 650 Harry Road, San Jose, CA 95120-6099, USA
}

\begin{abstract}
High-field electron spin resonance experiments have been performed on an Er fullerene extract, containing about $1 \%$ endohedral Er fullerenes $\left(\mathrm{Er}_{m} @ \mathrm{C}_{82}\right)$. The ESR spectra of $\left(\mathrm{Er}_{m} @ \mathrm{C}_{82}\right)$ show two resonances, which we propose to originate from the $\left(\mathrm{Er} @ \mathrm{C}_{82}\right)$ molecules, one resonance from an unpaired electron on the fullerene cage and one from the $\mathrm{Er}^{3+}$ 4f electrons.
\end{abstract}

\section{Introduction}

The possibility to encapsulate atoms inside closed carbon cages, first proposed by Kroto et al. [1], gives rise to a wide range of novel molecules and materials. Fullerene cage molecules containing metal atoms $\left(\mathbf{M} @ \mathbf{C}_{2 n}\right)$ [2], the so-called endohedral metallofullerenes, can nowadays successfully be prepared in macroscopic quantities. Characterization of these metallofullerenes in the laboratory-produced soot is done by mass spectrometry and electron spin resonance (ESR), the latter to obtain more specific information about the electronic structure of the metallofullerenes. Until now several metallofullerenes including La@ $\mathrm{C}_{82}[3-6], \mathrm{Y} @ \mathrm{C}_{82}[5,7,8]$, and $\mathrm{Sc} @ \mathrm{C}_{82}$ $[6,9]$ have been produced, extracted and characterized. One of the most interesting properties of these novel forms of molecules is that a charge transfer takes place between the fullerene cage and the encaged metal atoms. The metal atoms are suggested to be trivalent according to earlier experiments $[3,4]$, which means that a La atom

* Corresponding author. $\left(5 s^{2} 5 p^{6} 4 f^{0} 5 d^{1} 6 s^{2}\right)$ donates its two $6 s$ valence electrons as well as a $5 \mathrm{~d}$ electron to the $\mathrm{C}_{\mathbf{8 2}}$ fullerene cage. Two extra electrons on $\mathrm{C}_{82}$ form a closed shell $\mathrm{C}_{82}^{2-}$ cage (isoelectronic with $\mathrm{C}_{84}$ ) and leave a single unpaired electron on the fullerene cage. Here we will investigate another metal atom encaged in fullerenes, $\operatorname{Er}\left(5 s^{2} 5 p^{6} 4 f^{12} 6 s^{2}\right)$, which has an electronic structure comparable with a La atom.

\section{Material}

The soot containing the endohedral Er fullerenes $\left(\mathrm{Er}_{m} @ \mathrm{C}_{2 n}\right)$ is produced by arc-vaporization of cored carbon electrodes packed with Erbium oxides and graphite powder. This soot was extracted with $\mathrm{CS}_{2}$ and the resulting powder contained the stable endohedral $\mathrm{Er}$ fullerenes. Fig. 1 shows a time-of-flight mass spectrum of material which is laser desorbed and single-photon postionized from the dry powder, containing about $1 \%$ endohedral Er fullerenes. The mass spectrum thus obtained is a true abundance spectrum. Large peaks are present for both the dominant $\mathrm{C}_{60}$ and $\mathrm{C}_{70}$ clusters. The 


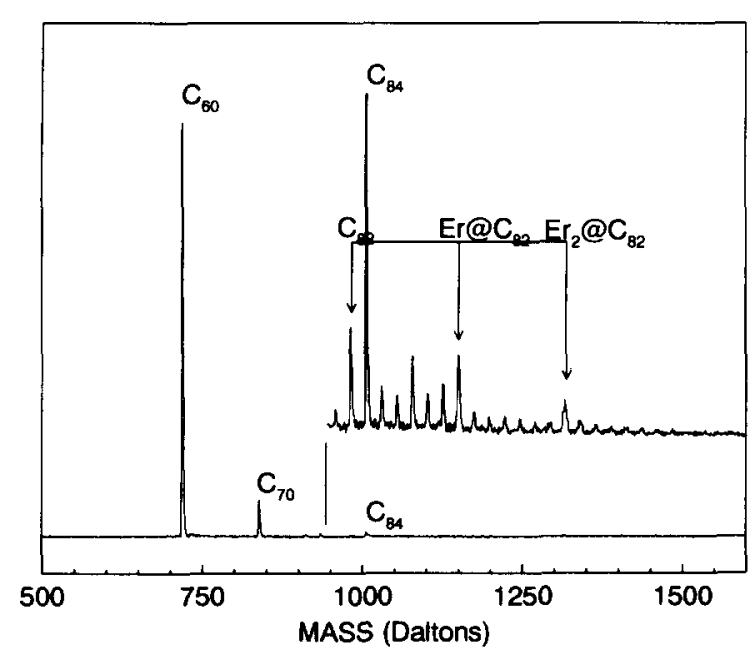

Fig. 1. Time-of-flight mass spectrum of material laser desorbed from the Er fullerene extract, containing about $1 \%$ endohedral Er fullerenes $\left(\mathrm{Er}_{m} @ \mathrm{C}_{82}\right)$. The inset shows the peaks of the endohedral Er fullerenes, $\mathrm{Er} @ \mathrm{C}_{82}$ and $\mathrm{Er}_{2} @ \mathrm{C}_{82}$.

inset of Fig. 1 shows the peaks corresponding to the endohedral Er fullerenes, coinciding with some smaller empty fullerene peaks. Together with the mono-metallofullerenes, Er@ $\mathrm{C}_{82}$, also some di-metallofullerenes, $\mathrm{Er}_{2} @ \mathrm{C}_{82}$, are present in the fullerene extract.

\section{Experimental setup}

For the high magnetic field electron spin resonance (ESR) experiments we use a millimeter wave vector network analyzer (MVNA) [10] as a source/detector for our prototype high field ESR spectrometer. Where conventional ESR experiments typically operate at a fixed frequency between 9 and $40 \mathrm{GHz}$ in fields upto $2 \mathrm{~T}$, we have the advantage of tunable frequencies ranging from 50 to $250 \mathrm{GHz}$, which can be locked to a cavity resonance. These high frequency ESR experiments are possible in magnetic fields upto $30 \mathrm{~T}$ and temperatures down to $1.2 \mathrm{~K}$, which makes our setup more sensitive to $g$-value anisotropies. The heterodyne detection technique we use has the advantage that we are able to measure both phase and amplitude of the signal simultaneously. With a combination of oversized waveguides and a mylar beamsplitter, high frequency radiation was coupled into a largely oversized resonant cylindrical cavity with a $Q$ factor of 15000 (empty) at $78 \mathrm{GHz}$, where the powder is put at the bottom of the cavity. At a certain frequency locked to a cavity resonance mode, a measurement of the reflected signal as a function of the external magnetic field then gives the complex ESR absorption spectrum.

\section{Results}

The ESR spectra of the endohedral Er fullerenes $\left(\mathrm{Er}_{m} @ \mathrm{C}_{82}\right.$ ) show two clear resonances. At high magnetic fields and for several different frequencies a relatively sharp resonance is found, corresponding to $g=2.005 \pm 0.001$. (See Fig. 2.) This resonance corresponds to transitions of an unpaired electron $\left(S=\frac{1}{2}\right)$ residing in an unoccupied molecular orbital (half-filled $\pi$ orbital) of the fullerene cage. In contrast to similar (low-field, low-frequency) ESR experiments on $\mathrm{La}_{m} @ \mathrm{C}_{82}[3,4,6], \mathrm{Y}_{m} @ \mathrm{C}_{82}[7,9]$ and $\mathrm{Sc}_{m} @ \mathrm{C}_{82}[6,9]$, we observe no indications of a hyperfine splitting in $\mathrm{Er}_{m} @ \mathrm{C}_{82}$, even not at low frequencies, although Er has a nuclear magnetic moment with spin $\frac{7}{2}$. This can be explained by the fact that the spin of the unpaired elec$\operatorname{tron}\left(S=\frac{1}{2}\right)$ is subject to spin-spin interactions with the $\mathrm{Er}^{3+} 4$ f electrons $(J=15 / 2)$, which leads to a broadening of the observed transition and to the lack of observable hyperfine splitting in the ESR spectra. This spin-spin interaction does not play a role in the other metallofullerene ESR spectra mentioned, since they lack the unpaired $4 \mathrm{f}$ electrons.

A second much broader resonance is found at lower magnetic fields with an apparent $g$-value of $\approx 8.6$ at $80 \mathrm{GHz}$, which can be seen in Fig. 3. We propose that this observed resonance originates from transitions of $\mathrm{Er}^{3+} 4 \mathrm{f}$ electrons $(J=15 / 2)$, because similar strongly anisotropic $g$-values are found for $\mathrm{Er}^{3+}$ ions incorporated in lanthanide ethylsulphates. This large $g$-value indicates a strong spin-orbit coupling like in the free $\mathrm{Er}^{3+}$ ion ground state, meaning an effective crystal field shielding. Contributions to this resonance can be given by the mono-metallofullerenes as well as the di-metallofullerenes present in the fullerene extract, both containing $\mathrm{Er}^{3+}$ ions. The broad line shape of this resonance can be explained by a spin-spin interaction between the $\mathrm{Er}^{3+} 4 \mathrm{f}$ electrons, and a strong spin-lattice coupling between the $\mathrm{Er}^{3+} 4 \mathrm{f}$ electrons and the fullerene cage, but until now we have no experimental evidence for this. Similar low field resonances cannot be observed for the earlier mentioned metallofullerenes, because the trivalent ions, $\mathrm{La}^{3+}, \mathrm{Y}^{3+}$ and $\mathrm{Sc}^{3+}$, have completely filled electron shells. The two resonances in the ESR spectra of $\mathbf{E r}_{m} @ \mathbf{C}_{82}$, one from an electron on the fullerene cage and one from the $\mathrm{Er}^{3+}$ ion, strongly suggest the electronic structure can be described by $\mathrm{Er}^{3+} @ \mathrm{C}_{82}^{3-}$. This means that the Er 6s electrons pair in the lowest unoccupied molecular orbital (LUMO) of the $\mathrm{C}_{82}$ fullerene, while one $\mathrm{Er} 4 \mathrm{f}$ electron occupies a higher molecular orbital and remains unpaired. 

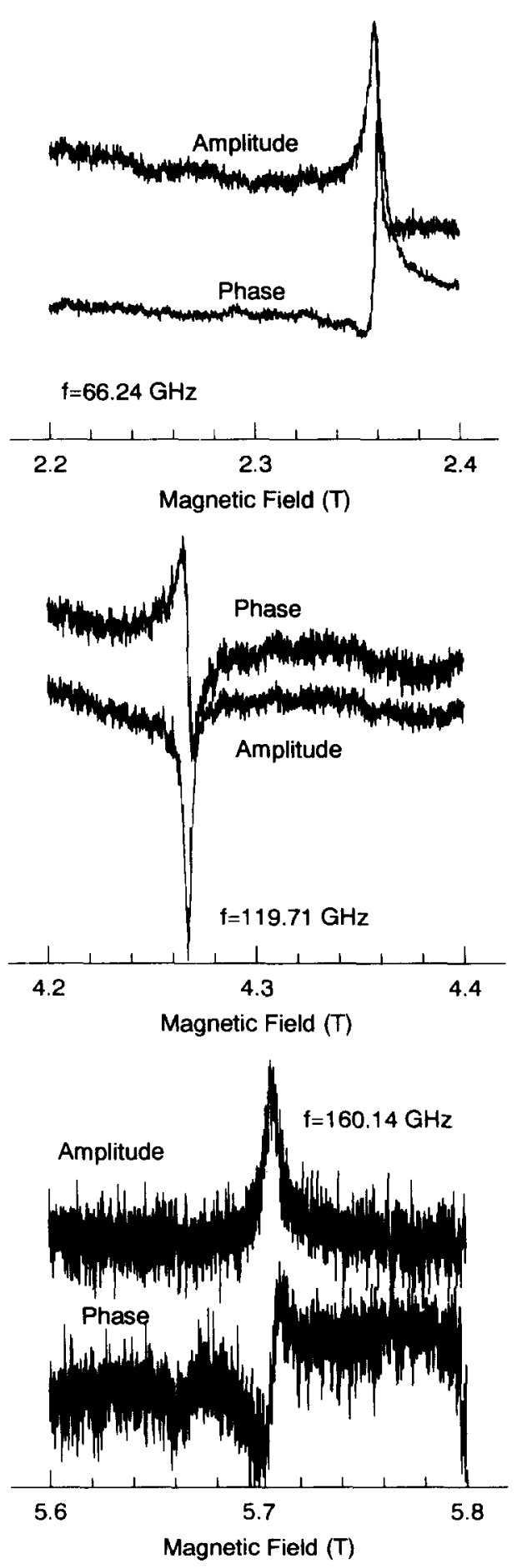

Fig. 2. Some typical ESR spectra of the Er fullerene extract $\left(\mathrm{Er}_{m} @ \mathrm{C}_{82}\right)$ for some different frequencies at $T=4.2 \mathrm{~K}$, corresponding to $g=2.005 \pm 0.001$.

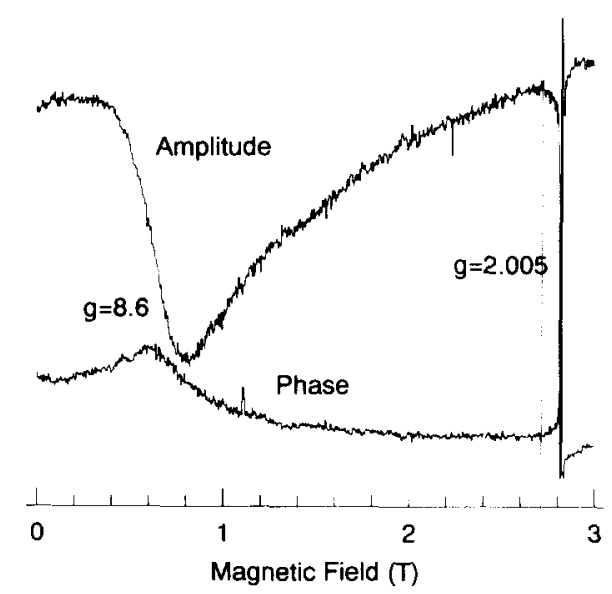

Fig. 3. ESR spectrum $(f=79.91 \mathrm{GHz})$ of the Er fullerene extract $\left(\mathrm{Er}_{m} @ \mathrm{C}_{82}\right)$ at $T=1.2 \mathrm{~K}$, where both resonances are visible.

\section{Summary}

We have developed and tested a prototype high field electron spin resonance spectrometer for the $50-250 \mathrm{GHz}$ frequency range, and used this to study the electronic structure of endohedral $\mathrm{Er}$ fullerenes $\left(\mathrm{Er}_{m} @ \mathrm{C}_{82}\right)$. We observed a resonance for $g=2.005$, showing that the fullerene cage contains one unpaired electron, donated by the Er atoms. The low field resonance reflects the $\mathrm{Er}^{3+}$ valence in this fullerene system, confirming the electronic structure of $\mathrm{Er} @ \mathrm{C}_{82}$ to be $\mathrm{Er}^{3+} @ \mathrm{C}_{82}^{3-}$. In contrast to $\mathrm{La}_{m} @ \mathrm{C}_{82}, \mathrm{Y}_{m} @ \mathrm{C}_{82}$ and $\mathrm{Sc}_{m} @ \mathrm{C}_{82}$, which have also a valency of $3+$, we find no indications of a hyperfine splitting in $\mathrm{Er}_{m} @ \mathrm{C}_{82}$.

\section{Acknowledgements}

This work is partially supported by the "Stichting voor Fundamenteel Onderzoek der Materie" (FOM) with the financial support from the "Nederlandse Organisatie voor Zuiver Wetenschappelijk Onderzoek" (ZWO). PvL gratefully acknowledges financial support from the Dutch Foundation for Scientific Research (NWO).

\section{References}

[1] H.W. Kroto, J.R. Heath, S.C. O'Brien, R.F. Curl and R.E. Smalley, Nature 318 (1985) 162.

[2] Y. Chai, T. Guo, C. Jin, R.E. Haufler, L.P.F. Chibante, J. Fure, L. Wang, J.M. Alford and R.E. Smalley, J. Phys. Chem 95 (1991) 7564. 
[3] R.D. Johnson, M.S. de Vries, J.R. Salem, D.S. Bethune and C.S. Yannoni, Nature 355 (1992) 239.

[4] K. Kikuchi, S. Suzuki, Y. Nakao, N. Nakahara, T. Wakabayashi, H. Shiromaru, K. Saito, I. Ikemoto and Y. Achiba, Chem. Phys. Lett. 216 (1993) 67.

[5] M. Hoinkis, C.S. Yannoni, D.S. Bethune, J.R. Salem, R.D. Johnson, M.S. Crowder and M.S. de Vries, Chem. Phys. Lett. 198 (1992) 461.

[6] D.S. Bethune, C.S. Yannoni, M. Hoinkis, M.S. de Vries, J.R. Salem, M.S. Crowder and R.D. Johnson, Z. Phys. D 26 (1993) 153.

[7] H. Shinohara, H. Sato, Y. Saito, M. Ohkohchi and Y. Ando, J. Phys. Chem. 96 (1992) 3571.
[8] J.H. Weaver, Y. Chai, G.H. Kroll, C. Jin, T.R. Ohno, R.E. Haufler, T. Guo, J.M. Alford, J. Conceicao, L.P.F. Chibante, A. Jain, G. Palmer and R.E. Smalley, Chem. Phys. Lett. 190 (1992) 460.

[9] S. Suzuki, S. Kawata, H. Shinomura, K. Yamauchi, K. Kikuchi, T. Kato and Y. Achiba, J. Phys. Chem. 96 (1992) 7159.

[10] P. Goy, M. Gross and J.M. Raimond, in: Proc. 15th Int. Conf. on IR and mm Waves, Orlando, FL, ed. R.J. Temkin (1990) p. 172. 